\title{
Recommendation for cholecystectomy protocol based on intraoperative ultrasound - a single-centre retrospective case-control study
}

\author{
Maciej Sebastian, Jerzy Rudnicki \\ Department of General, Minimally Invasive and Endocrine Surgery, Wroclaw Medical University, Wroclaw, Poland
}

Videosurgery Miniinv 2021; 16 (1): 54-61

DOI: https://doi.org/10.5114/wiitm.2020.93999

\begin{abstract}
Introduction: There is a strong need to make laparoscopic cholecystectomy as safe as possible, but sometimes complications in the form of bile duct and/or vascular injury occur. The safe plane of dissection can be precisely identified with intraoperative ultrasound, ensuring reduction of the complication rate to a minimum.

Aim: To evaluate the advantages of the cholecystectomy protocol based on the use of intraoperative ultrasound during laparoscopic and open cholecystectomy.

Material and methods: The study group consisted of 700 patients with symptomatic cholecystolithiasis, which was divided into two subgroups: with the critical view of safety only (312 patients) and with the critical view of safety + laparoscopic/open cholecystectomy ultrasound (388 patients). Laparoscopic cholecystectomy and conversion in patients from the second subgroup were performed under the control of intraoperative ultrasound.

Results: We did not observe any biliary complications, and the visualization of the common bile duct, the proper hepatic artery and the portal vein was obtained in every patient from the critical view of safety + laparoscopic/open cholecystectomy ultrasound group. The mean time of the operation was significantly shorter and the conversion, biliary injury and intraoperative bleeding rates were significantly lower in this group of patients.

Conclusions: Intraoperative ultrasound is a very efficient and safe method of guidance, and its use should be standard along with the critical view of safety during cholecystectomy.
\end{abstract}

Key words: cholecystectomy, ultrasonography, laparoscopy, bile ducts.

\section{Introduction}

A protocol for cholecystectomy should guide the surgeon throughout the procedure and give answers during critical steps to avoid disastrous complications. The most dangerous moment of the operation is preparation in the region of the hepatoduodenal ligament; thus the identification of the right plane of dissection is crucial. The critical view of safety may not be enough during severe inflammatory changes in the region of Calot's tri- angle $[1,2]$. Cholangiography is time consuming, cumbersome and does not visualize the vascular structures $[3,4]$.

\begin{abstract}
Aim
Our goal is to show the advantages of intraoperative ultrasound during laparoscopic or open cholecystectomy and to emphasize the utility and flexibility of these methods of visualization. Introducing our protocol into everyday practice may diminish the
\end{abstract}


rate of conversions and complications during cholecystectomy.

\section{Material and methods}

The study group consisted of 700 (382 women and 318 men) patients and was divided into two subgroups. The first subgroup consisted of 312 patients (168 women and 144 men) and was operated on between January 2010 and June 2014. The second group consisted of 388 patients (214 women and 174 men) and was operated on between July 2014 and June 2018 in one department of surgery. In the first subgroup (CVS only group) the only method of intraoperative visualization was the critical view of safety (CVS); in the second subgroup the CVS and laparoscopic ultrasound (LUS) (CVS + LUS group) and open cholecystectomy ultrasound (OUS) (Open + OUS group) were used. Operations with LUS started in July 2015 and from then on all patients were operated under LUS guidance. The inclusion criterion for the study was symptomatic cholecystolithiasis. The exclusion criterion was pre- or postoperatively diagnosed cancer of the gallbladder (4 $(0.57 \%)$ patients were postoperatively excluded due to gallbladder cancer), preoperative acute cholecystitis and previous operations in the abdominal cavity. Our aim was to obtain a maximally homogeneous group for statistical analysis without any additional intraoperative factors which a priori change the surgical situs. In the group with CVS only, laparoscopic cholecystectomy (LC) was performed in 289 patients and conversion (Open group) in 23 (7.4\%) patients. In the group with CVS + LUS, LC was performed in 378 patients and conversions in 10 (2.6\%) patients. Written informed consent was obtained from all patients before surgery. All procedures were in accordance with the ethical standards of the 1964 Declaration of Helsinki and its later amendments.

Cholecystectomies were performed on an elective basis by three surgeons experienced in LC/open cholecystectomy (OC) (> 150 cholecystectomies) and LUS/OUS (> 70 examinations). We used the laparoscopic probe Toshiba PEF-704 LA (frequency 7.0 MHz) for LUS and the micro probe Toshiba PVF-745V (frequency $7.0 \mathrm{MHz}$ ) for OUS and the diagnostic ultrasound system Toshiba NemioMX SSA-590A, all manufactured in Japan. LUS and/or OUS were performed routinely in every patient in the CVS + LUS and OUS groups. A laparoscopic ultrasound probe was insert- ed through the epigastric $10 \mathrm{~mm}$ (transverse view) or umbilical $10 \mathrm{~mm}$ trocar (longitudinal view). OUS was performed through the laparotomy wound. Vascular and avascular structures were differentiated with duplex Doppler function. LUS and/or OUS were performed before, during and after preparation in Calot's triangle whenever it was needed. A sodium chloride isotonic solution was used to improve acoustic coupling between the probe and the scanned surface whenever it was needed. When the inflammatory plane was hard to prepare there was an attempt to perform fundus-first, subtotal cholecystectomy under the control of LUS, and when it was impossible the operation was converted and OUS was used to find the right plane of dissection. When safe preparation could not be achieved, subtotal cholecystectomy or cholecystostomy was performed. The key structure which was visualized to proceed further with preparation and clipping was the "Mickey Mouse sign" a characteristic configuration of the bile duct, the proper hepatic artery and the portal vein in the hepatoduodenal ligament which is similar to the head of Mickey Mouse (Photos 1 A, B). There was also a possibility to visualize a "four-tube sign" - a connection of the cystic duct with the common bile duct (CBD) (Photo $1 \mathrm{C}$ ). The hyperechoic stents in the bile duct usually made the identification easier and whenever possible they were left in situ for the duration of surgery and removed thereafter in a planned manner (Photo $1 \mathrm{D}$ ). The high rate of patients with stents in the bile ducts 29 (7.5\%) in the CVS + LUS/OUS group was associated with the fact that our department is the regional centre for endoscopic retrograde cholangiopancreatography (ERCP) and after this procedure the patients choose our department for LC. The indications for bile duct stenting before the operation were choledocholithiasis with narrowing of the bile ducts. Of course, ERCP is not a standard procedure before LC but undoubtedly hyperechoic stents are easily found with LUS/OUS. The decisions of further steps during the operation were based on a protocol for cholecystectomy based on intraoperative ultrasound (Figure 1).

\section{Statistical analysis}

Statistical analysis included the unpaired $t$-test or Mann-Whitney $U$ test for continuous and Fisher's exact test for binary variables. The level of statistical significance was set at 95\% $(p<0.05)$. 

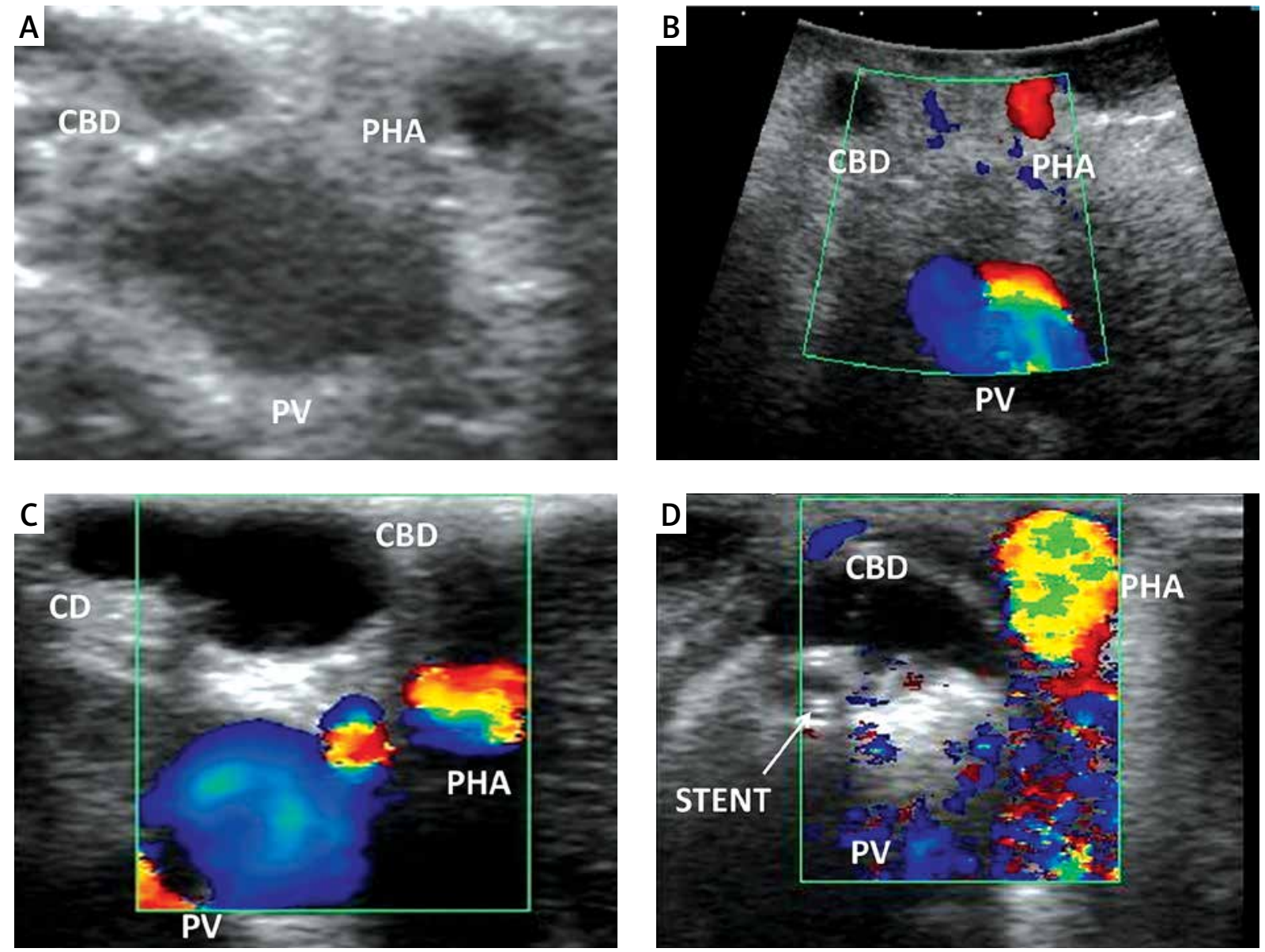

Photo 1. A - "Mickey Mouse” sign - LUS. B - Mickey Mouse" sign - OUS. C - “Four-tube sign”. D - Hyperechoic stent in the CBD

$C A$ - cystic artery, CBD - common bile duct, CD - cystic duct, LUS - laparoscopic ultrasound, OUS - open cholecystectomy ultrasound, PHA - proper hepatic artery, PV-portal vein.

\section{Results}

The mean time of LC in the group with CVS + LUS was $58.2 \mathrm{~min}$ and LUS $1.7 \mathrm{~min}(2.9 \%$ of the time of the whole procedure) and it was together significantly shorter than in the group with CVS only. There were no significant differences between the two subgroups of patients according to the age, length of hospital stay and time of open cholecystectomy (Table I). The conversion, biliary injury and intraoperative bleeding rates were significantly lower in the group with CVS + LUS (Table II). Conversion was under control firstly by LUS and then by OUS. The reason for conversion in the CVS only group was in $4(1.4 \%)$ patients biliary injury, in $6(2.1 \%)$ patients intraoperative bleeding and in 13 (4.5\%) patients chronic inflammation without safe plane of dissection. In the CVS + LUS group the reason for conver- sion was in $2(0.5 \%)$ patients intraoperative bleeding and in $8(2 \%)$ patients chronic inflammation without a safe plane of dissection. The bleeding was stopped intraoperatively in both groups of patients and did not cause any postoperative long-term complications. LUS and/or OUS were completed in $100 \%$ of patients with visualization of the CBD, proper hepatic artery and portal vein. We did not observe any biliary complications and the rate of intraoperative bleeding was significantly lower in the CVS + LUS group in comparison to the CVS only group. The use of LUS/OUS during the operation defined in most of the cases the proper plane of dissection, which was associated with safe performance of the procedure and a significantly lower rate of conversions. The availability of LUS enabled the performance of a significantly higher rate of subtotal cholecystectomies, which was associated with the ultrasonographically 


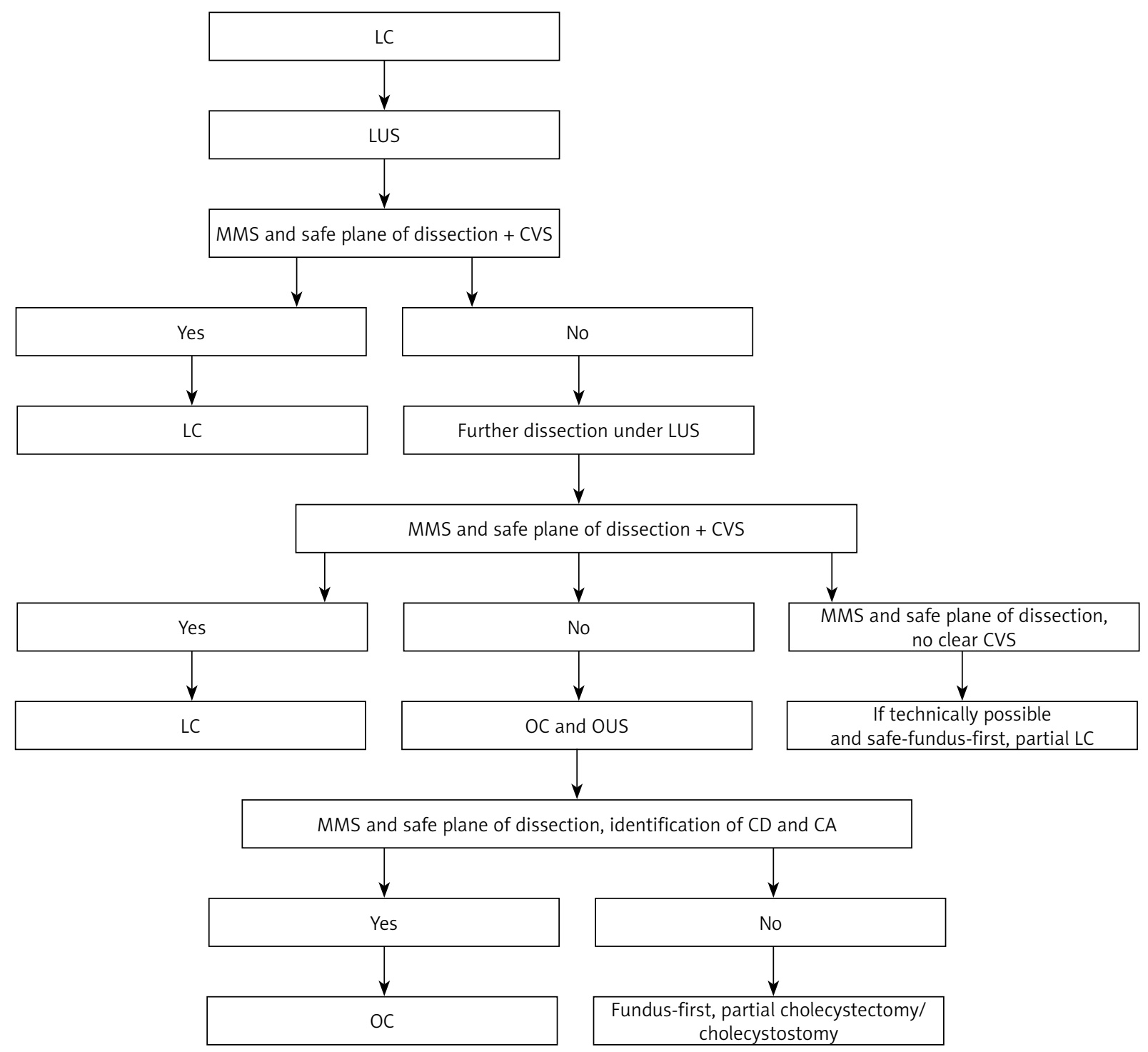

Figure 1. Protocol for cholecystectomy based on intraoperative ultrasound

CBD - common bile duct, CVS - critical view of safety, LC - laparoscopic cholecystectomy, LUS - laparoscopic cholecystectomy ultrasound, MMS - Mickey Mouse sign, OC - open cholecystectomy, OUS - open cholecystectomy ultrasound.

defined safe plane of dissection. We did not observe any statistically significant differences between the groups where conversion and open cholecystectomies were performed.

\section{Discussion}

LC remains the gold standard in the treatment of symptomatic cholecystolithiasis $[5,6]$. Bile duct injury (BDI) during OC occurs in $0.1-0.3 \%$ of cases, in $\mathrm{LC}$ varies between 0.08 and $0.5 \%$ and it reaches the rates of $\mathrm{OC}$, which may be associated with bet- ter technique, improved equipment and overcoming the "learning curve" [7]. The main reason for BDI is misperception, not the lack of skill, inadequate knowledge or misjudgement [8]. There are several methods introduced to avoid BDI. The most common and reliable visual method is the CVS introduced by Strasberg in 1995, which includes preparation of the lower third of the gallbladder out from the liver, cleaning the region of Calot's triangle (through the established window we should see the $V$ segment of the liver); the cystic duct and the cystic artery should be the only visible ductal structures which are left 

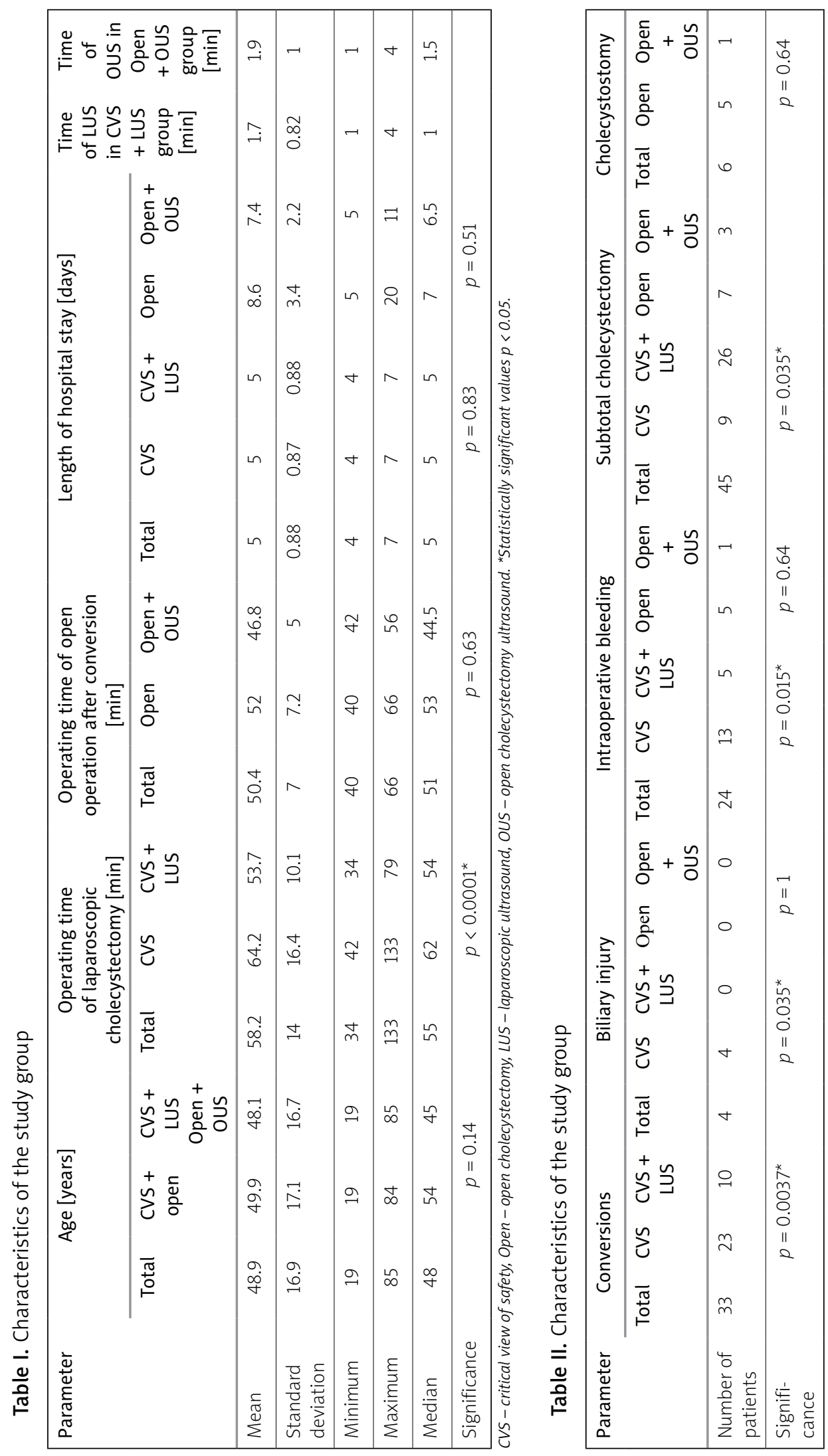
between the gallbladder and the CBD [1, 2]. Another, older method of visual identification is the infundibular technique - the junction between the gallbladder and the cystic duct should be visualized. In the case of inflammation relying solely on this method may lead to BDI and it should be abandoned as the only method of visualization [9]. Routine intraoperative cholangiography (IOC) remains a controversial issue and a growing number of surgeons are not performing it because BDIs are not preventable in $100 \%$ of cases and IOC does not visualize the blood vessels in the hepatoduodenal ligament [3, 4]. Pesce et al. stated that LC can be performed safely with low rates of biliary complications without the use of IOC and that the most important factors for safe LC are accurate preoperative evaluation of clinical risk factors, precise operative procedures and conversion to an open approach in doubtful cases [10]. Another problem with IOC may be the misinterpretation of the view, resulting in CBD cannulation and injury requiring conversion, repair over T-tube or reconstruction [9].

Near-infrared fluorescence cholangiography (NIRF-C) is a sensitive and safe method of biliary tree visualization. The fluorophore substance indocyanine green administered intravenously is eliminated with the bile and fluorescence is detected with an infrared camera with no adverse effects of such a procedure. NIRF-C does not visualize the vascular structures the same as IOC but it gives information about the biliary tree before and during preparation. To conclude, further studies are needed to evaluate this method and its more frequent use in the future [11-13].

The importance of proper vascular identification is associated with the increased rate of vascular injuries concomitant with BDI [5]. When it occurs, usually the right hepatic artery and a bile duct are injured (about $90 \%$ of vasculobiliary injuries) leading to biliary ischaemia (possible bile leakage and stenosis), but hepatic ischaemia is uncommon. The typical scenario of extreme vasculobiliary injuries (BDI and the proper hepatic artery, the common hepatic artery, the main portal vein or the right portal vein) was the LC converted to an open procedure due to severe chronic or acute inflammation. The fundus-down cholecystectomy was attempted and severe bleeding occurred, resulting in many cases with hepatic infarction and right hepatectomy or even orthotopic liver transplant. The death rate in these cases was $50 \%$ [14]. There is also a recommenda- tion that the possible vascular injury associated with the BDI should be excluded with the CT angiography prior to any repair [5]. Thus, the surgeon should, instead of cholecystectomy, perform cholecystostomy or subtotal cholecystectomy without the hazardous preparation [14]. The proper line of dissection will show the OUS.

There exist recommendations of safe LC [11, 15]. The highest impact on the successful procedure is observed for CVS and dome-down (fundus-first)/ partial cholecystectomy as possible alternative techniques. Any difficulties with obtaining the CVS should force the surgeon to use another method of visualization such as IOC/LUS, perform conversion or get help from a senior surgeon skilled in hepatobiliary (HPB) surgery [11]. A study performed on 394 surgeons revealed that CVS was not identified correctly by $75 \%$ descriptively and by $21 \%$ visually, and in reality only $27 \%$ used the CVS technique and $56 \%$ of the surgeons used the non-recommended infundibular method. Routine IOC was used by $16 \%$ and LUS by $<1 \%[6]$. When confronted with difficult anatomy $76.7 \%$ of surgeons preferred IOC, $16 \%$ converted, $6.4 \%$ would consult with a senior surgeon and only $0.8 \%$ chose LUS. The results of this study stressed the need of education or re-education of surgeons on safe biliary surgery and more common use of LUS [6]. The CVS as the most important factor of a safe LC is recognized by $93 \%$ of HPB surgeons but only by $76 \%$ of general surgeons, which is the next reason to promote further education on this topic [11]. The CVS is also one part of the Safe Cholecystectomy Program (SCP) introduced by the Society of American Gastrointestinal and Endoscopic Surgeons (SAGES) [16]. Bogacki et al. evaluated the SCP among 524 European surgeons (265 from Denmark and 259 from Poland) of whom 184 filled in the previously prepared survey completely. The usefulness of each of six SAGES SCP rules was high. Rules number two (consider intra-operative time-out) and six (get help from another surgeon) were considered more important by surgical residents, while rule number three (understand potential for aberrant anatomy) was considered more important by surgical specialists. All of these moments of doubt and especially rule number four (use of cholangiography or other methods of visualization) and five (recognize dangerous zone) may be assisted by the use of LUS as many times as it is needed until the solution is found. Also this survey confirmed a low knowl- 
edge of the SCP among European surgeons: $21.4 \%$ of residents and $42.1 \%$ of specialists were aware of implementation of these rules in the clinical setting, which of course is the indication for education and re-education of the SAGES SCP rules [17].

LUS and OUS are techniques which are non-invasive and non-irradiating, can be performed before, during and after dissection and repeated as many times as needed without a negative impact on the patient and operating staff. Their use should be routine during $\mathrm{LC}$ to get the experience needed in difficult cases when the anatomy is obscured [18, 19]. Unfortunately not many surgeons are using this method, possibly due to the long learning curve and time consumed at the beginning of the learning process, but throughout the time it is getting faster without a huge impact on the whole procedure (5-10 min for LUS vs. 10-17 min for IOC) [20]. LUS can visualize the biliary anatomy in $98-100 \%$ of cases without BDI mainly due to its repeatability, avoidance of biliary tree preparation and cannulation, control on one monitor with a laparoscopic view and detection of blood vessels [2, 21-23]. A big advantage of LUS and OUS is the Doppler function, which enables differentiation between vascular and avascular structures [18]. The potential limitation of ultrasound is the inability to find the exact location of injury; thus IOC is a better technique in such a complication [20]. Detailed mapping of the biliary anatomy cannot be achieved with LUS, but it is not needed during $\mathrm{LC}$ - the most important factor is the safe plane of dissection [19]. The documented conversion rate from LC to OC is $5 \%$ and LUS may diminish the number of conversions in $5.9-91 \%$ of patients [21, 24, 25], but if it occurs the operation can be safely continued with the OUS and the decision on a possible subtotal cholecystectomy/cholecystostomy based on OUS.

\section{Conclusions}

The protocol of cholecystectomy based on intraoperative LUS and OUS enables the surgeon to perform the laparoscopic/open cholecystectomy in a safe way without bile duct and vascular injury. The probes for LUS and OUS are lying on one table and switching between these two methods is very flexible. We agree that CVS is a crucial method of a safe LC with LUS and OUS enabling the safe plane of dissection especially when the anatomy is obscured and obtaining a clear CVS is limited [26]. Thus, these techniques should be more widely used and surgical residents should be taught LUS and OUS, as is stressed by other authors [11, 19]. We recommend the use of LUS/OUS in every cholecystectomy because it does not take too much time, with clear identification of key structures. Undoubtedly the lack of LUS would have forced the surgeons to convert more frequently and the possibility of complications would have been higher. The limitation of our study was a relatively small study group, especially the group where conversion and open cholecystectomy was performed; thus further studies including larger groups of patients in more than one surgical centre are needed in order to strengthen our findings.

\section{Conflict of interest}

The authors declare no conflict of interest.

\section{References}

1. Buddingh KT, Nieuwenhuijs VB, van Buuren L, et al. Intraoperative assessment of biliary anatomy for prevention of bile duct injury: a review of current and future patient safety interventions. Surg Endosc 2011; 25: 2449-61.

2. Strasberg SM, Hertl M, Soper NJ. An analysis of the problem of biliary injury during laparoscopic cholecystectomy. Am Coll Surg 1995; 180: 101-25.

3. Aziz O, Ashrafian H, Jones C, et al. Laparoscopic ultrasonography versus intra-operative cholangiogram for the detection of common bile duct stones during laparoscopic cholecystectomy: a meta-analysis of diagnostic accuracy. Int J Surg 2014; 12 : $712-9$

4. Machi J, Oishi AJ, Tajiri T, et al. Routine laparoscopic ultrasound can significantly reduce the need for selective intraoperative cholangiography during cholecystectomy. Surg Endosc 2007; 21: $270-4$

5. Hogan NM, Dorcaratto D, Hogan AM, et al. latrogenic common bile duct injuries: increasing complexity in the laparoscopic era: a prospective cohort study. Int J Surg 2016; 33: 151-6.

6. Daly SC, Deziel DJ, Li X, et al. Current practices in biliary surgery: do we practice what we teach? Surg Endosc 2016; 30: 3345-50.

7. Halbert C, Pagkratis S, Yang J, et al. Beyond the learning curve: incidence of bile duct injuries following laparoscopic cholecystectomy normalize to open in the modern era. Surg Endosc 2016; 30: 2239-43.

8. Way LW, Stewart L, Gantert W, et al. Causes and prevention of laparoscopic bile duct injuries: analysis of 252 cases from a human factors and cognitive psychology perspective. Ann Surg 2003; 237: 460-9.

9. Strasberg SM, Eagon CJ, Drebin JA. The "hidden cystic duct" syndrome and the infundibular technique of laparoscopic cholecystectomy: the danger of the false infundibulum. J Am Coll Surg 2000; 191: 661-7. 
10. Pesce A, Portale TR, Minutolo V, et al. Bile duct injury during laparoscopic cholecystectomy without intraoperative cholangiography: a retrospective study on 1,100 selected patients. Dig Surg 2012; 29: 310-4.

11. Conrad C, Wakabayashi G, Asbun HJ, et al. IRCAD recommendation on safe laparoscopic cholecystectomy. J Hepatobiliary Pancreat Sci 2017; 24: 603-61.

12. Ishizawa T, Bandai Y, Ijichi M, et al. Fluorescent cholangiography illuminating the biliary tree during laparoscopic cholecystectomy. Br J Surg 2010; 97: 1369-77.

13. Pesce A, Piccolo G, La Greca G. Utility of fluorescent cholangiography during laparoscopic cholecystectomy: a systematic review. World J Gastroenterol 2015; 21: 7877-83.

14. Strasberg SM, Gouma DJ. 'Extreme' vasculobiliary injuries: association with fundus-down cholecystectomy in severely inflamed gallbladders. HPB (Oxford) 2012; 14: 1-8.

15. Hori T, Oike F, Furuyama H, et al. Protocol for laparoscopic cholecystectomy: is it rocket science? World I Gastroenterol 2016; 22: 10287-303.

16. SAGES (2014) The sages safe cholecystectomy program. http:// www.sages.org/safe-cholecystectomy-program/10.

17. Bogacki P, Krzak J, Gotfryd-Bugajska K, et al. Evaluation of the usefulness of the SAGES Safe Cholecystectomy Program from the viewpoint of the European surgeon. Videosurgery Miniinv 2020; 15: 80-6.

18. Dili A, Bertrand C. Laparoscopic ultrasonography as an alternative to intraoperative cholangiography during laparoscopic cholecystectomy. World J Gastroenterol 2017; 23: 5438-50.

19. Deziel DJ. Laparoscopic ultrasound and cholecystectomy: a small but vital parade. Laparosc Surg 2018; 2: 8.

20. Ishido K, Hakamada K, Machi J. Laparoscopic ultrasound during laparoscopic cholecystectomy - a systematic review. Ann Emerg Surg 2017; 2: 1020.

21. Gwinn EC, Daly S, Deziel DJ. The use of laparoscopic ultrasound in difficult cholecystectomy cases significantly decreases morbidity. Surgery 2013; 154: 909-15.

22. Li JW, Feng B, Wu L, et al. Intraoperative cholangiography in combination with laparoscopic ultrasonography for the detection of occult choledocholithiasis. Med Sci Monit 2009; 15: 126-30.

23. Buanes T, Waage A, Mjåland O, et al. Bile leak after cholecystectomy significance and treatment: results from the National Norwegian Cholecystectomy Registry. Int Surg 1996; 81: 276-9.

24. Ballal M, David G, Willmott S. Conversion after laparoscopic cholecystectomy in England. Surg Endosc 2009; 23: 2338-44.

25. Machi J, Johnson JO, Deziel DJ, et al. The routine use of laparoscopic ultrasound decreases bile duct injury: a multicenter study. Surg Endosc 2009; 23: 384-8.

26. Pesce A, Portale TR, Di Stefano B. Clinical value of intra-operative ultrasonography during laparoscopic cholecystectomy. Laparosc Surg 2018; 2: 7.

Received: 15.12.2019, accepted: 24.02.2020. 